\title{
Breakdown of Small Diameter Si Nanowires under External Electric Field: First-principle Calculation
}

\author{
R.Q. Zhang, N. Gao, Z. Wen*, and Q. Jiang* \\ Key Laboratory of Automobile Materials (Jilin University), Ministry of Education, and \\ School of Materials Science and Engineering, Jilin University, Changchun, 130022, \\ China
}

\begin{abstract}
The electronic and structural properties of [111] SiNWs with a diameter range of $D=0.66-1.89 \mathrm{~nm}$ under the external electric field along axial direction are calculated using first-principle calculation. With the external electric field applied, energy band gap $E_{\mathrm{g}}(D, F)$ decreases and finally closes, as $F$ increases. The closure strength of external electric field $F_{\text {closure }}$ decreases with increasing $D$. HOMO, LUMO and Mulliken charge analysis are shown to interpret the effect of external electric field on the electronic properties of SiNWs. The bond length and angle of $\mathrm{Si}$ atoms vary with increasing $F$. Further increasing of $F$ results in breakdown of the geometry structure, which is size-dependent.
\end{abstract}

\section{Introduction}

Silicon nanowires (SiNWs) have attracted more and more attention because of its interface compatibility with existing silicon-based technology and satisfaction for the need of high integration in ultra-large-scale integration circuits. ${ }^{1}$ Since bulk-quantity SiNWs were grown by laser ablation method, ${ }^{2,3}$ the study of SiNWs is developed rapidly, and it has wide implications, such as field effect transistors, ${ }^{4-8}$ photodetectors, ${ }^{9,} 10$ sensors, ${ }^{11-13}$ field emission devices, ${ }^{14}, 15$ photovoltaic cells, ${ }^{16}$ logic gates $^{17}$ and thermoelectricity materials. ${ }^{18,19}$ With the device miniaturization, the size of SiNWs will further decrease. Recently, small diameter SiNWs have been synthesized with various methods. ${ }^{20-23}$

It is inevitable that the nanodevices would be under an external or self-produced electric field in application and the field would affect the electrical and structural properties of SiNWs, which suggests that the investigation of the properties of SiNWs under external electric field is necessary. The external electric field can induce deformation of semiconductor material, and according to classical physics when the electric field is large enough the insulating system will be breakdown. With the scanning tunneling microscope (STM), by combining a strong electric field formed between the STM tip and the surface, Si atoms can be transferred from the surface to the tip and re-deposited on a predetermined surface site. ${ }^{24}$ Under an external electric field, the highest occupied molecular orbital (HOMO) and the lowest unoccupied molecular orbital (LUMO) energies of silica nanowires (NWs) decrease with different speed and finally cross, that is breakdown of these NWs. ${ }^{25}$ The external electric field also causes axial electrostrictive deformation in single carbon nanotubes, ${ }^{26}$ tunes electron-phonon coupling in graphene ${ }^{27}$ and affects the atomic structure of $\mathrm{Cu}$ nanowires. ${ }^{28}$

\footnotetext{
* Corresponding author. Fax: 86-431-85095876; Email: wenzi@jlu.edu.cn (Z. Wen), jiangq@jlu.edu.cn (Q. Jiang)
} 
The small diameter SiNW-based high-performance electronic and optoelectronic devices have been required to obtain increasingly strict and well-defined performance. With the diameter of SiNWs decreases into several nanometers, a detailed control at the atomic level is needed. All of these could be realized under the help of computer simulations. As an important means of investigation of material science, the computer simulation has been used wildly and received substantial success. It is an efficiency method to connect the structures and properties of materials. The functions of computer simulation are as follows: a) compare the academic results that getting from computer simulation and from experiment, b) analyze and explain the reasons that are still unknown in experiment or theory, c) predict new phenomenon and properties before experiment, d) predict properties that are difficultly realized in experiment or in ideal conditions.

In this contribution, we calculate the energy band gap $E_{g}(D, F)$, where $D$ and $F$ denote diameter of SiNWs and the strength of external electric field respectively, and structural variations of small diameters $(D=0.66-1.89 \mathrm{~nm})$ [111] SiNWs by using Density Functional Theory (DFT). High $F$ induces $E_{g}(D, F)$ closure and structure breakdown of SiNWs. The results, introduced and illustrated here, suggest the importance of the external electric field in affecting the properties of SiNWs.

\section{Calculation methodology}

Four kinds $\mathrm{SiNWs}$ of $\mathrm{Si}_{14} \mathrm{H}_{18}, \mathrm{Si}_{38} \mathrm{H}_{30}, \mathrm{Si}_{74} \mathrm{H}_{42}$ and $\mathrm{Si}_{122} \mathrm{H}_{54} \mathrm{NW}$ are studied in this contribution. Each of the considered SiNW structures was placed in the super cell with a vacuum space at least $10 \AA$ outside the wire at each lateral direction, enough to eliminate the influence of NWs in neighbor cells and the wire axial direction was infinitely extended. The unit cell of [111] SiNWs consists of six (110) atomic planes and the cross-section of SiNWs is a regular hexagon. $D$ of the SiNWs is defined by calculating the diameter of the circular shape with the same area as each NW, which is $0.66,1.07$, 1.47 and $1.89 \mathrm{~nm}$ for $\mathrm{Si}_{14} \mathrm{H}_{18}, \mathrm{Si}_{38} \mathrm{H}_{30}, \mathrm{Si}_{74} \mathrm{H}_{42}$ and $\mathrm{Si}_{122} \mathrm{H}_{54} \mathrm{NW}$, respectively. The dangling bonds of the surface $\mathrm{Si}$ atoms are saturated by $\mathrm{H}$. The detail information can be found in our previous report. ${ }^{29}$ The structure of $\mathrm{Si}_{38} \mathrm{H}_{30} \mathrm{NW}$ with $D=1.07 \mathrm{~nm}$ is shown in Figure 1 as representation.
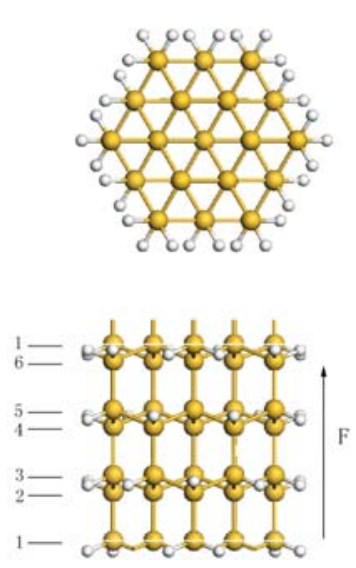

Figure 1. Top and side views of the ball and stick structures of $\mathrm{Si}_{38} \mathrm{H}_{30}$ unit cell are given. The big and small balls denote $\mathrm{Si}$ and $\mathrm{H}$ atoms respectively. The number $1 \sim 6$ shows different Si layers in the unit cell. The arrow shows the direction of the external electric field. 
The properties of [111] SiNWs are calculated using DFT, ${ }^{30,31}$ which is implemented in $\mathrm{DMol}^{3}$ model. ${ }^{32,33}$ The generalized gradient approximation (GGA) functional with the PW91 method ${ }^{34}$ was employed as the exchange-correlation functional. All Electron Relativitistic (AER), ${ }^{35,36}$ which includes all core electrons explicitly and introduces some relativistic effects into the core, was used for core treatment. In addition, Double Numerical plus Polarization (DNP) ${ }^{32}$ was chosen as the basis set with orbital cutoff of 4.6 $\AA$. We use smearing techniques ${ }^{37}$ to achieve self-consistent field convergence with a smearing value of $0.005 \mathrm{Ha}(1 \mathrm{Ha}=27.2114 \mathrm{eV})$. The structure of SiNWs was then relaxed using the delocalized internal coordinate optimization scheme. The convergence tolerance for energy of $1.0 \times 10^{-5} \mathrm{Ha}$, maximum force of $0.002 \mathrm{Ha} / \AA$, and maximum displacement of $0.005 \AA$ in the geometry optimization are taken. Our previous report ${ }^{29}$ has justified the validity of the above simulation techniques, meanwhile the discussions between DFT and GW (a combination of the Green function $G$ and the screened Coulomb interaction $W)^{38}$ calculations can also be seen there.

The external electric field was directly applied along $<111>$ direction with $F=0.00$, $0.25,0.50,0.75,1.00 \mathrm{~V} / \AA$, respectively and later geometry relaxation was carried out. Larger $F$ is applied until geometry structure of SiNWs breakdown.

\section{Results and discussion}

$E_{\mathrm{g}}(D, F)$ function with $D$ and $F$ is shown in Figure 2. In absence of $F$, as $D$ increase, $E_{g}(D, 0)$ decrease, which is consistent with reported size-dependence of the energy band gap. ${ }^{39-42}$ With $F=0.25 \mathrm{~V} / \AA$ applied, $E_{\mathrm{g}}(D, F)$ change a little compared with that in absence of $F$. The drop tendencies of $E_{\mathrm{g}}(D, F)$ with $D$ at any $F$ are similar. However, as the $D$ increasing the variation of $E_{\mathrm{g}}(D, F)$ reduces. Take $F=0.50 \mathrm{~V} / \AA$ for example, the variation of $E_{\mathrm{g}}(D, F)$ are $68.5 \%$ and $50.4 \%$ for $D$ increasing from 0.66 to $1.07 \mathrm{~nm}$ and from 1.47 to $1.89 \mathrm{~nm}$ respectively. In other words, $E_{\mathrm{g}}(D, F)$ and the variation are size-dependent.

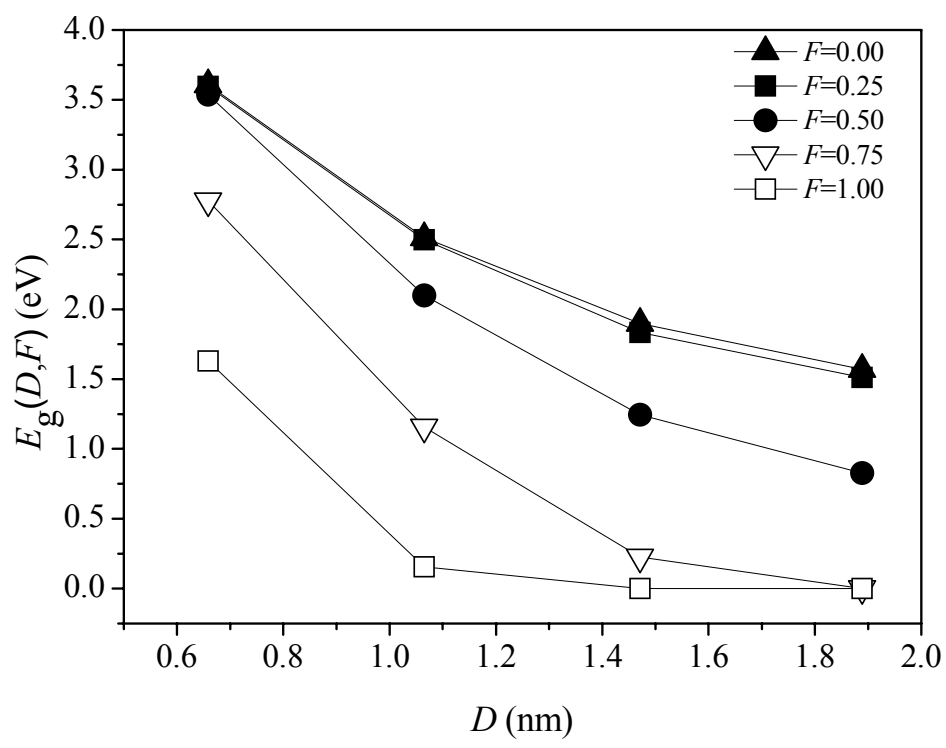

Figure 2. $E_{\mathrm{g}}(D, F)$ functions of SiNWs for $\mathrm{F}=0.00,0.25,0.50,0.75$ and $1.00 \mathrm{~V} / \AA$ of $\mathrm{Si}_{14} \mathrm{H}_{18}, \mathrm{Si}_{38} \mathrm{H}_{30}, \mathrm{Si}_{74} \mathrm{H}_{42}$ and $\mathrm{Si}_{122} \mathrm{H}_{54} \mathrm{NW}$. The straight lines are drawn as a guide to the eyes. 
For a constant $D, E_{\mathrm{g}}(D, F)$ decreases with $F$ increasing, which is similar with the situation for silica NWs. ${ }^{43}$ It is found that for $\mathrm{Si}_{74} \mathrm{H}_{42}$ and $\mathrm{Si}_{122} \mathrm{H}_{54} \mathrm{NW}, F=1$ and $0.75 \mathrm{~V} / \AA$ result in closure of $E_{\mathrm{g}}(D, F)$ respectively, as shown in Figure 2. It is expected that there is a certain $F$ can bring out the closure of $E_{\mathrm{g}}(D, F)$ for $\mathrm{Si}_{14} \mathrm{H}_{18}$ and $\mathrm{Si}_{38} \mathrm{H}_{30}$ NW. The closure strength of external electric field $F_{\text {closure }}$ are calculated, which are listed in TABLE I. It is found that $F_{\text {closure }}$ increase with the decrease of $D$. Note that we check carefully for $F_{\text {closure, }}$ and that of $\mathrm{Si}_{74} \mathrm{H}_{42} \mathrm{NW}$ is $0.83 \mathrm{~V} / \AA$ rather than $1 \mathrm{~V} / \AA$. Compared with silica NWs, ${ }^{25}$

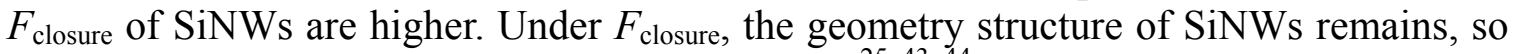
$F_{\text {closure }}$ is classified as a soft breakdown of $E_{\mathrm{g}}(D, F) .^{25,43,44}$

TABLE I. The closure field and broken-down field of different diameter of [111] SiNWs

\begin{tabular}{cccc}
\hline Stoichiometry & $\mathbf{D}(\mathbf{n m})$ & $\boldsymbol{F}_{\text {closure }}(\mathbf{V} / \mathbf{\AA})$ & $\boldsymbol{F}_{\text {break }}(\mathbf{V} / \mathbf{\AA})$ \\
\hline $\mathrm{Si}_{14} \mathrm{H}_{18}$ & 0.66 & 1.29 & 1.37 \\
$\mathrm{Si}_{38} \mathrm{H}_{30}$ & 1.07 & 1.05 & 1.41 \\
$\mathrm{Si}_{74} \mathrm{H}_{42}$ & 1.47 & 0.83 & 1.10 \\
$\mathrm{Si}_{122} \mathrm{H}_{54}$ & 1.89 & 0.75 & 1.21 \\
\hline
\end{tabular}

Further increasing $F$, the geometry structure of SiNWs will break down. The breakdown strength of external electric field $F_{\text {break }}$ of SiNWs is also listed in TABLE I. As expected, $F_{\text {break }}$ is larger than $F_{\text {closure }}$ and it is also size-dependent.

In the following, the effect of external electric field on the electronic and structural properties of SiNWs is studied in detail. It is known that the HOMO and LUMO energy is important for understanding and predicting the electronic properties and behaviours of nanostructures. ${ }^{25,43}$ In TABLE II, the HOMO and LUMO energies with $F=0.25 \mathrm{~V} / \AA$, compared with in absence of $F$, are shown for the different SiNWs. It is found that with $F$ applied, both the HOMO and LUMO energies of SiNW decrease, but at different speed. So when $F$ is large enough, the two decrease lines must cross, that is the breakdown of $E_{\mathrm{g}}(D, F)$ for SiNWs, corresponding to $F_{\text {closure. }}$ Size-dependence of HOMO and LUMO energies, as shown in TABLE II, brings out the size-dependence of $E_{\mathrm{g}}(D, F)$.

TABLE II The HOMO and LUMO energies in $\mathrm{eV}$ of SiNWs with and without $F$ in $\mathrm{V} / \AA$

\begin{tabular}{ccccc}
\hline \multirow{2}{*}{ Stoichiometry } & \multicolumn{2}{c}{ HOMO } & \multicolumn{2}{c}{ LUMO } \\
\cline { 2 - 5 } & $\boldsymbol{F = 0 . 0 0}$ & $\boldsymbol{F}=\mathbf{0 . 2 5}$ & $\boldsymbol{F}=\mathbf{0 . 0 0}$ & $\boldsymbol{F}=\mathbf{0 . 2 5}$ \\
\hline $\mathrm{Si}_{14} \mathrm{H}_{18}$ & -6.055 & -6.150 & -2.178 & -2.306 \\
$\mathrm{Si}_{38} \mathrm{H}_{30}$ & -5.502 & -5.596 & -2.990 & -3.095 \\
$\mathrm{Si}_{74} \mathrm{H}_{42}$ & -5.267 & -5.356 & -3.365 & -3.463 \\
$\mathrm{Si}_{122} \mathrm{H}_{54}$ & -5.136 & -5.226 & -3.559 & -3.656 \\
\hline
\end{tabular}

Mulliken charge analysis is calculated, charge distributions of $\mathrm{Si}_{38} \mathrm{H}_{30} \mathrm{NW}$ are given in Figure 4. Mulliken charges of all 6 layers are redistributed under the external electric field. Let $\mathrm{L}_{i}$ denote $i$-th layer of $\mathrm{Si}_{38} \mathrm{H}_{30} \mathrm{NW}$, and the order of $i$-th layer is shown in Figure 1. As $F$ increase, the charges of $\mathrm{L}_{1}, \mathrm{~L}_{3}$ and $\mathrm{L}_{6}$ increase, while those of $\mathrm{L}_{2}, \mathrm{~L}_{4}$ and $\mathrm{L}_{5}$ decrease. This suggests that $F$ results in inhomogeneity of the charge distribution. It is found that charges of $\mathrm{L}_{1}, \mathrm{~L}_{2}, \mathrm{~L}_{5}, \mathrm{~L}_{6}$ change little under $F$. However, charges of $\mathrm{L}_{3}$ and $\mathrm{L}_{4}$ vary largely. The electron transfer occurs between these tow layers. The external electric field "pushes" positive charge along its direction. It means that electrons of $\mathrm{L}_{4}$ transfer to $\mathrm{L}_{3}$. Then charges of $\mathrm{L}_{3}$ increase while that of $\mathrm{L}_{4}$ decrease under $F$. Higher $F$ induces more electron transfer. 


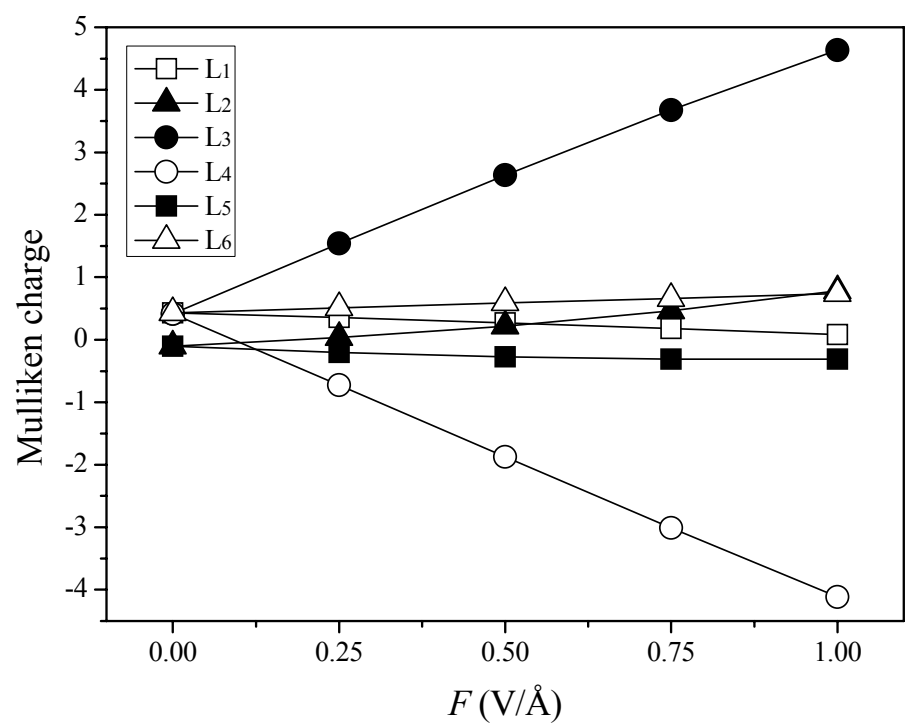

Figure 4. Mulliken charge distribution of different layers for $\mathrm{Si}_{38} \mathrm{H}_{30} \mathrm{NW}$.

The external electric field, which is similar to a mechanical field, should deform atomic structures of SiNWs with atomic movements. We study the geometry information by determining the layer distance $L$ and bond angle $\theta$ of $\mathrm{Si}$ atoms. Let $L_{i-j}$ denote the distance between $i$ and $j$ layer of SiNWs. Layer distance $L(F)$ for $\mathrm{Si}_{38} \mathrm{H}_{30} \mathrm{NW}$ under $F$ are listed in TABLE III. As $F$ increases, $L_{1-2}, L_{3-4}, L_{4-5}, L_{5-6}, L_{6-1}$ increase, while $L_{2-3}$ has an opposite trend.

TABLE III. Layer distance function $L(F)$ in $\AA$ for $\mathrm{Si}_{38} \mathrm{H}_{30} \mathrm{NW}$ with $F$ in V/Å. $\Delta$ is the percent difference between $F=1$ and $F=0$.

\begin{tabular}{cccccccc}
\hline $\boldsymbol{L}$ & $\boldsymbol{F}=\mathbf{0 . 0 0}$ & $\boldsymbol{F}=\mathbf{0 . 2 5}$ & $\boldsymbol{F}=\mathbf{0 . 5 0}$ & $\boldsymbol{F = 0 . 7 5}$ & $\boldsymbol{F}=\mathbf{1 . 0 0}$ & $\boldsymbol{F = 1 . 4 1}$ & $\boldsymbol{\Delta}(\mathbf{\%})$ \\
\hline$L_{1-2}$ & 2.361 & 2.364 & 2.369 & 2.376 & 2.388 & 2.422 & 1.14 \\
$L_{2-3}$ & 0.809 & 0.768 & 0.721 & 0.665 & 0.599 & 0.439 & -25.96 \\
$L_{3-4}$ & 2.360 & 2.362 & 2.368 & 2.379 & 2.392 & 2.411 & 1.36 \\
$L_{4-5}$ & 0.809 & 0.845 & 0.878 & 0.909 & 0.939 & 1.049 & 16.07 \\
$L_{5-6}$ & 2.361 & 2.360 & 2.360 & 2.361 & 2.364 & 2.366 & 0.13 \\
$L_{6-1}$ & 0.796 & 0.798 & 0.802 & 0.811 & 0.824 & 0.892 & 3.52 \\
\hline
\end{tabular}

The relative change $\Delta$ is larger for $L_{2-3}$ and $L_{4-5}$ while it is only several percent for the others, particularly $\Delta_{5-6}=0.13 \% . L_{2-3}$ and $L_{4-5}$ have the largest contractive and elongated deformations of $-25.96 \%$ and $16.07 \%$ respectively. The relative change $\Delta$ for $\mathrm{Si}_{38} \mathrm{H}_{30}$ is larger than this for $\mathrm{Si}_{14} \mathrm{H}_{18}$ which is given in our previous paper. ${ }^{29}$ This suggests that $\Delta$ is size-dependent. As $F$ increase, the variation of $L$ increase, so the deformation amounts of NWs increase.

$\theta$ distributions under $F=0.00,0.50,1.00$ and $1.41 \mathrm{~V} / \AA$ are given in Figure 5. As $F$ increase, the distribution range of $\theta$ becomes larger. In absence of $F, \theta$ distributes almost in the range from $105^{\circ}$ to $114^{\circ}$, and the average value is $109.5^{\circ}$ which is $\mathrm{Si}$ atoms angle in the bulk Si. When $F=1 \mathrm{~V} / \AA$, two peaks appear in the distribution of bond angles, and the distribution is from about $90^{\circ}$ to $130^{\circ}$. As shown in Figure 5, before breakdown of geometry structure, the distribution of angles of NWs is more dispersed, as $F$ increase. 


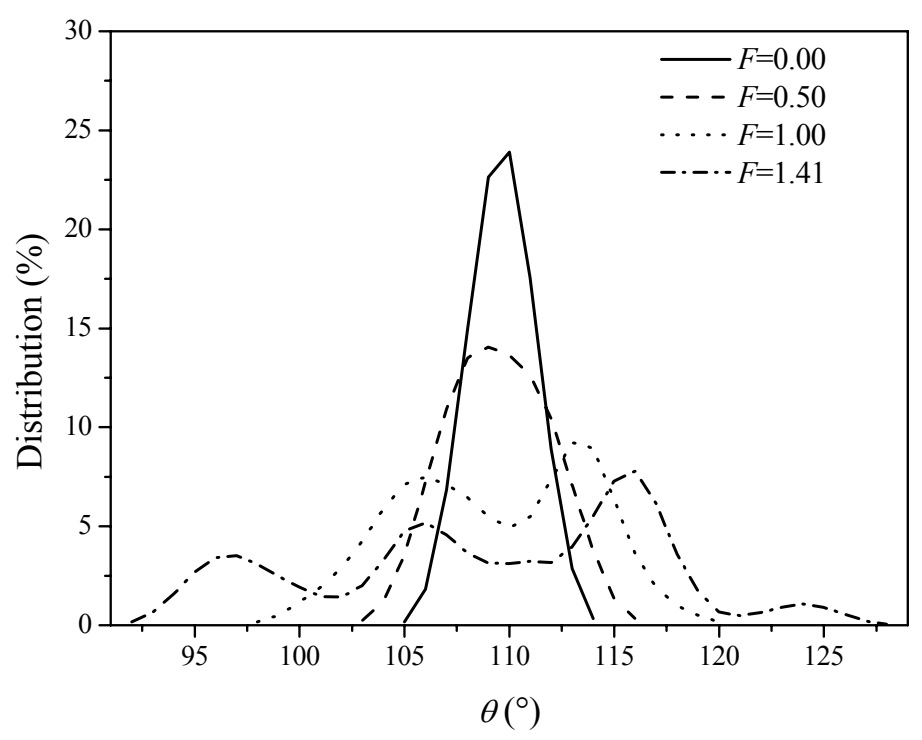

Figure 5. Distribution of Si-Si-Si angles for $<111>\mathrm{Si}_{38} \mathrm{H}_{30} \mathrm{NWs}$ with $F$.

As $F$ increase, $L$ and $\theta$ vary, which certainly bring out the variation of the bond length $l$ of $\mathrm{Si}$ atoms. Figure 6 present $l$ distribution of $\mathrm{Si}_{38} \mathrm{H}_{30} \mathrm{NW}$ under different $F$. Without $F, l$ of $\mathrm{Si}_{38} \mathrm{H}_{30}$ distribute from $2.354 \AA$ to $2.369 \AA$, which is contractive compared with bulk of $2.369 \AA$ due to the one dimension structure. As $F$ increase, $l$ elongates and the distribution is more dispersed.

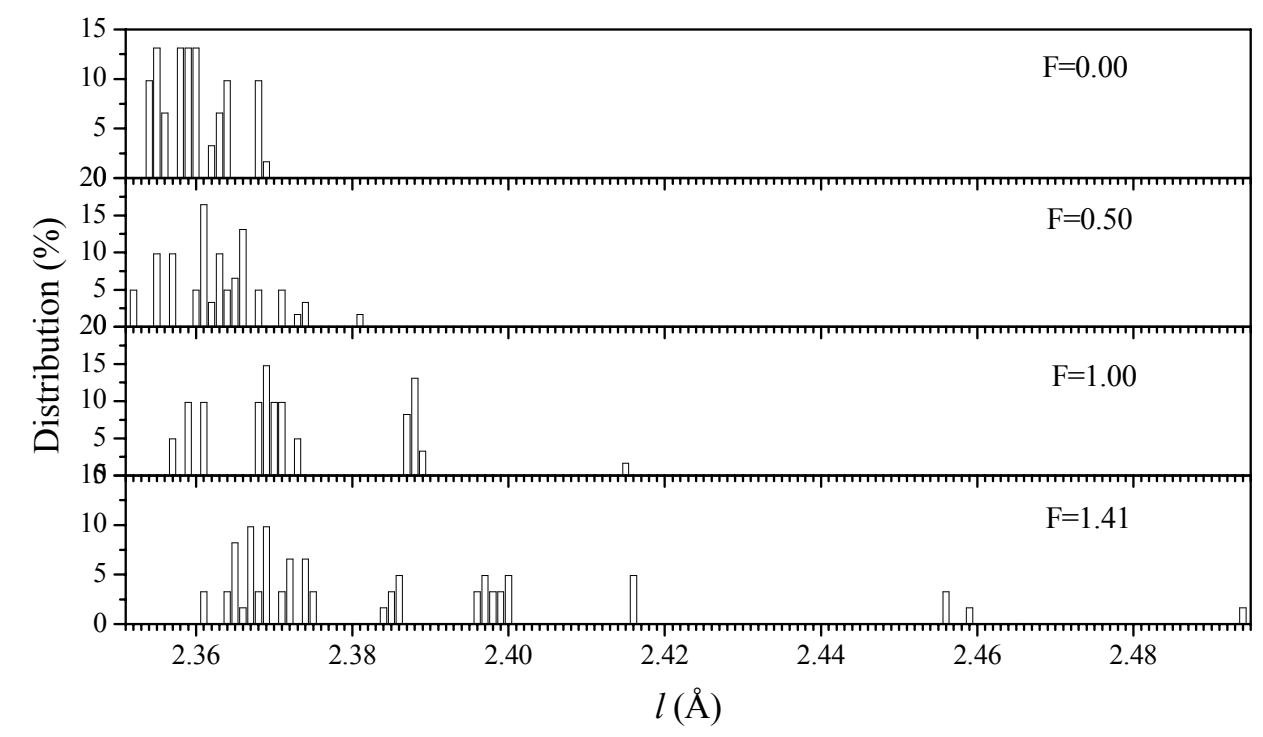

Figure 6. Distribution of Si-Si bond lengths $l$ for $\mathrm{Si}_{38} \mathrm{H}_{30} \mathrm{NW}$ applied with $F$.

In the last, the structure of $\mathrm{Si}_{38} \mathrm{H}_{30} \mathrm{NW}$ under $F_{\text {break }}$ is discussed based TABLE III, Figure 5 and 6 . The structure have deformed heavily under $F_{\text {break }} . L\left(F_{\text {break }}\right)$ functions, which are listed in TABLE III, are obtained from the average axial coordinates of layer Si atoms because the layer atoms appear no longer in the same layer strictly. The 
deformation enhanced compared with $F=1 \mathrm{~V} / \AA$. This is confirmed from the more dispersed distribution of $\theta$ and $l$ in Figure 5 and $6 . \theta$ and $l$ distribute in the range from $92^{\circ}$ to $128^{\circ}$ and from 2.361 to $2.494 \AA$ under $F=1.41 \mathrm{~V} / \AA$. When $F$ reaches larger than 1.41 $\mathrm{V} / \AA$, the atomic structure of $\mathrm{Si}_{38} \mathrm{H}_{30} \mathrm{NWs}$ is breakdown and there is no more a stable geometry structure due to the existence of non-convergence of energy.

\section{Conclusion}

In summary, $E_{\mathrm{g}}(D, F)$ functions of [111] SiNWs are calculated and the breakdown of SiNWs under external electric field is studied using first-principle calculation. The results show that $E_{\mathrm{g}}(D, F)$ decreases with $F$ and $D$ increasing, and SiNWs will be subject to breakdown in the strong electric field, including electronic and structural properties. $F_{\text {closure }}$ of $E_{\mathrm{g}}(D, F)$ decreases remarkably with $D$ increasing. Both bond angle and bond length vary under $F$, and as $F$ increases the variations increase. These findings from present calculation will provide useful information for failure of SiNWs under external electric field in the application of nanodevices.

\section{Acknowledgements}

The authors acknowledge support by National Key Basic Research and Development Program (Grant No. 2004CB619301) and NNSFC (Grant No. 50525204).

\section{Reference}

1. P. S. Peercy, Nature 406, 1023 (2000).

2. A. M. Morales and C. M. Lieber, Science 279, 208 (1998).

3. Y. F. Zhang, Y. H. Tang, N. Wang, D. P. Yu, C. S. Lee, I. Bello and S. T. Lee, Appl. Phys. Lett. 72, 1835 (1998).

4. M. Kobayashi and T. Hiramoto, J. Appl. Phys. 103 , 053709 (2008).

5. P. Zhang, T. S. Mayer and T. N. Jackson, ACS Nano 1, 6 (2007).

6. J. Xiang, W. Lu, Y. Hu, Y. Wu, H. Yan and C. M. Lieber, Nature 441, 489 (2006).

7. Y. Cui, Z. Zhong, D. Wang, W. U. Wang and C. M. Lieber, Nano Lett. 3, 149(2003).

8. J. Goldberger, A. I. Hochbaum, R. Fan and P. Yang, Nano Lett. 6, 973 (2006).

9. C. Yang, C. J. Barrelet, F. Capasso and C. M. Lieber, Nano Lett. 6, 2929 (2006).

10. A. Zhang, S. You, C. Soci, Y. Liu, D. Wang and Y.-H. Lo, Appl. Phys. Lett. 93, 121110 (2008).

11. I. Y. Park, Z. Y. Li, X. M. Li, A. P. Pisano and R. S. Williams, Biosens. Bioelectron. 22, 2065 (2007).

12. Y. Cui, Q. Wei, H. Park and C. M. Lieber, Science 293, 1289 (2001).

13. C.-H. Lin, C.-Y. Hsiao, C.-H. Hung, Y.-R. Lo, C.-C. Lee, C.-J. Su, H.-C. Lin, F.-H. Ko, T.-Y. Huang and Y.-S. Yang, Chem. Commun. (44), 5749 (2008).

14. J. C. She, S. Z. Deng, N. S. Xu, R. H. Yao and J. Chen, Appl. Phys. Lett. 88, $013112(2006)$.

15. C. S. Chang, S. Chattopadhyay, L. C. Chen, K. H. Chen, C. W. Chen, Y. F. Chen, R. Collazo and Z. Sitar, Phys. Rev. B 68, 125322 (2003).

16. J. A. Martinez, N. Misra, Y. Wang, P. Stroeve, C. P. Grigoropoulos and A. Noy, Nano Lett. 0 (0) (2009).

17. B. A. Sheriff, D. Wang, J. R. Heath and J. N. Kurtin, ACS Nano 2, 1789 (2008). 
18. T. T. M. Vo, A. J. Williamson, V. Lordi and G. Galli, Nano Lett. 8, 1111 (2008).

19. A. I. Hochbaum, R. Chen, R. D. Delgado, W. Liang, E. C. Garnett, M. Najarian, A. Majumdar and P. Yang, Nature 451, 163 (2008).

20. Y. L. S. T. L. R.-Q. Zhang, Adv. Mater. 15, 635 (2003).

21. Kook-Nyung Lee, Suk-Won Jung, Kyu-Sik Shin, Won-Hyo Kim, Min-Ho Lee and W.-K. Seong, Small, 4, 642 (2008).

22. C. Wiethoff, F. M. Ross, M. Copel, M. Horn-von Hoegen and F.-J. Meyer zu Heringdorf, Nano Lett., 8, 3065 (2008).

23. N. R. B. Coleman, M. A. Morris, T. R. Spalding and J. D. Holmes, J. Am. Chem. Soc., 123, 187 (2001).

24. I.-W. Lyo and P. Avouris, Science, 253, 173 (1991).

25. Q. Sun, Q. Wang, Y. Kawazoe and P. Jena, Nanotechnology, 15, 260 (2004).

26. W. Guo and Y. Guo, Phys. Rev. Lett., 91, 115501 (2003).

27. J. Yan, Y. Zhang, P. Kim and A. Pinczuk, Phys. Rev. Lett., 98, 166802 (2007).

28. C. He, P. Zhang, Y. F. Zhu and Q. Jiang, J. Phys. Chem. C, 112, 9045 (2008).

29. R. Q. Zhang, W. T. Zheng and Q. Jiang, submitted (2009).

30. W. Kohn and L. J. Sham, Phys. Rev., 140, A1133 (1965).

31. P. Hohenberg and W. Kohn, Phys. Rev., 136, B864 (1964).

32. B. Delley, J. Chem. Phys., 113, 7756 (2000).

33. B. Delley, J. Chem. Phys., 92, 508 (1990).

34. J. P. Perdew and Y. Wang, Phys. Rev. B, 45, 13244 (1992).

35. M. Douglas and N. M. Kroll, Ann. Phys., 82, 89 (1974).

36. D. D. Koelling and B. N. Harmon, J. Phys. C, 10, 3107 (1977).

37. B. Delley, in Theoretical and Computational Chemistry, J. M. Seminario and P. Politzer, Editors, Vol. 2, p. 221-254, Elsevier, (1995).

38. F. Aryasetiawan and O. Gunnarsson, Rep. Prog. Phys., 61, 237 (1998).

39. B. Delley and E. F. Steigmeier, Appl. Phys. Lett., 67, 2370 (1995).

40. M.-F. Ng, L. Zhou, S.-W. Yang, L. Y. Sim, V. B. C. Tan and P. Wu, Phys. Rev. B, 76, 155435 (2007).

41. J.-A. Yan, L. Yang and M. Y. Chou, Phys. Rev. B, 76, 115319 (2007).

42. X. Zhao, C. M. Wei, L. Yang and M. Y. Chou, Phys. Rev. Lett., 92, 236805 (2004).

43. D. Zhang, G. Guo, C. Liu and R. Q. Zhang, J. Phys. Chem. B, 110, 23633 (2006).

44. T. Pompl, C. Engel, H. Wurzer and M. Kerber, Microelectron. Reliab., 41, 543 (2001). 